\section{LETTERS TO THE EDITORS}

The Editors do not hold themselves responsible for opinions expressed by their correspondents. No notice is taken of anonymous communications.

\section{Effective Magnetic Meridian for Cosmic Rays}

RECENT latitude surveys of cosmic-ray intensity made with neutron intensity monitors appear to confirm an earlier suspicion that the dipole approximation to the Earth's magnetic field fails to account accurately for the observed distribution of cosmic rays over the Earth's surface ${ }^{1-3}$. This has been revealed as a result of the much greater latitude variation observed with these instruments compared with charged-particle detectors. The LemaitreVallarta theory of the geomagnetic effect predicts a minimum in the cosmic-ray intensity coincident with the geomagnetic equator. Simpson et al. ${ }^{2}$ have collected the available observations of the equatorial minimum and conclude that the 'cosmic ray equator' fits more accurately the equator of a dipole rotated about the Earth's axis $40^{\circ}-45^{\circ}$ to the west of the 'best fit' dipole. Rothwell and Quenby ${ }^{3}$, on the other hand, find the magnetic dip equator fits adequately, and suggest that the Earth's real field rather than the dipole approximation aceounts for the observed distribution.

New evidence can be obtained from a determination of the 'cosmic-ray meridian' or line on the Earth's surface along which the rate of change of cosmic-ray intensity is greatest. Depending on which theory proves most acceptable, this might be expected to coincide with the geomagnetic meridian, the meridian of Simpson's rotated dipole, or with the magnetic declination. A determination of the cosmic-ray meridian through Melbourne has been carried out at the University of Tasmania using a monitor counting the disintegration-product neutrons from the local production of stars in lead. The monitor uses two counters and follows closely the design proposed by Simpson. It was installed in a Lincoln bomber provided for the work by the Royal Australian Air Force.

The cosmic-ray meridian was determined by flying the aircraft in a triangular circuit with a vertex at Melbourne (lat. $38^{\circ}$ S., long. $145^{\circ}$ E., geomagnetic lat. $47^{\circ} \mathrm{S}$.) and the base to the north. The aircraft flew at a pressure altitude of $20,000 \mathrm{ft}$. and completed the circuit twice on June 4, 1957.

If $\theta$ is the bearing of the cosmic-ray meridian and $r$ the rate of change of intensity along the meridian then $\theta$ and $r$ are related to $\theta_{1}$ and $r_{1}$, the bearing of the first leg of the flight and rate of change along the leg respectively, by the equation : $r_{1}=r \cos \left(\theta-\theta_{1}\right)$.

From the six legs, six independent equations in the two unknowns $r$ and $\theta$ were obtained. The method of least squares gave the following best values for $r$ and $\theta: \quad r=2.4 \pm 0.2$ per cent deg. ${ }^{-1}$ latitude; $\theta=10^{\circ} \pm 4^{\circ}$, east of north. The errors are standard errors.

This value of $\theta$ can be compared with the following magnetic data for Melbourne: magnetic declination, $10^{\circ}$ east of north ; geomagnetic meridian, $9 \cdot 5^{\circ}$ east of north; meridian of dipole rotated $45^{\circ}$ to west, $14 \cdot 5^{\circ}$ east of north.

It is clearly not possible to choose between the magnetic declination and the geomagnetic meridian; but
Table 1

\begin{tabular}{|l|c|c|c|}
\hline & $\begin{array}{c}\text { Magnetic } \\
\text { declination }\end{array}$ & $\begin{array}{c}\text { Geomagnetic } \\
\text { declination }\end{array}$ & $\begin{array}{c}\text { Rotated geomagnetic } \\
\text { dipolo declination }\end{array}$ \\
\hline Melbourne & $+10^{\circ}$ & $+9 \cdot 5^{\circ}$ & $+14 \cdot 5^{\circ}$ \\
Rome & $\pm 2 \cdot 5$ & -16 & $-14 \cdot 5$ \\
Istanbul & \pm 2 & -15 & $-8 \cdot 5$ \\
$\begin{array}{l}\text { Cape Town } \\
80^{\circ} \text { W., } 50^{\circ} \mathrm{S} .\end{array}$ & $+24 \cdot 5$ & +14 & -12 \\
\hline
\end{tabular}

Declinations to the east of north are positive.

the experiment suggests the rotated dipole theory may be wrong. It is unfortunate that nowhere in Australia does the difference between the declina. tion and the geomagnetic meridian exceed $2^{\circ}$, and it will be necessary to repeat the experiment elsewhere in order to distinguish between the rival theories. In order to obtain a sufficient rate of change of intensity with latitude, observations should be made between geomagnetic latitudes $30^{\circ}$ and $50^{\circ}$ north or south. Within these bands suitable localities can be found in the northern hemisphere over the Mediterranean, while Cape Town seems promising in the southern hemisphere. Even more suitable, but rather inaccessible, is the region to the west of the south tip of South America, represented by the point $\left(90^{\circ} \mathrm{W} ., 50^{\circ} \mathrm{S}.\right)$ in Table 1 , which compares the merits of selected localities.

The experiment described here seems capable of providing new evidence on the influence of the Earth's magnetic field on cosmic rays, and although it cannot be fruitfully pursued in Australia there is every reason to expect valuable results if it is performed in more suitable localities.

J. R. STOREY

A. G. FENTON

K. G. MCCRACKEN

Physics Department,

University of Tasmania, Hobart, Tasmania.

Rose, D. C., Fenton, K. B., Katzman, J., and Simpson, J. A., Canad. J. Phys., 34, 968 (1956).

${ }^{2}$ Simpson, J. A., Fenton, K. B., Katman, J., and Rose, D. C., Phys. Rev., 102,1648 (1956).

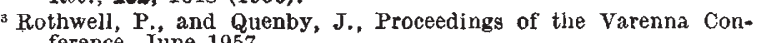
ference, June 1957.

\section{Single-Hop Propagation of Radio Waves to a Distance of $5,300 \mathrm{~km}$.}

In has been established that radio waves can be propagated between Ottawa in Canada and Slough in England by a single ionospheric reflexion. The one-hop mode of propagation is seen on the accom. panying ionogram (Fig. 1) at frequencies between 27 and 33 Mc./s. This ionogram was recorded at 1800 G.M.T., September 11, 1957. The low-angle part of the one-hop mode is intercepted by the Earth. The high-angle portion is associated with reflexion at apparent heights of 600 to more than $900 \mathrm{~km}$. The other traces correspond to two- and three-hop propagation.

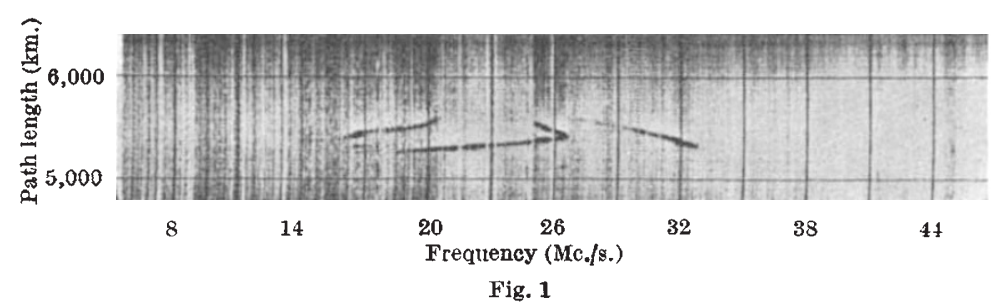

Fig. 1 